\title{
Improving anterior deltoid activity in a musculoskeletal shoulder model - an analysis of the torque-feasible space at the sternoclavicular joint
}

\author{
David Ingram $^{\mathrm{a} *}$, Christoph Engelhardt ${ }^{\mathrm{a}}$, Alain Farron ${ }^{\mathrm{b}}$, Alexandre Terrier ${ }^{\mathrm{a}}$ and Philippe Müllhaupt ${ }^{\mathrm{a}}$ \\ ${ }^{a}$ Ecole Polytechnique Fédérale de Lausanne, Switzerland; ${ }^{b}$ University Hospital Centre and University of Lausanne, Switzerland
}

(Received 27 January 2015; accepted 15 April 2015)

\begin{abstract}
Modelling the shoulder's musculature is challenging given its mechanical and geometric complexity. The use of the ideal fibre model to represent a muscle's line of action cannot always faithfully represent the mechanical effect of each muscle, leading to considerable differences between model-estimated and in vivo measured muscle activity. While the musculotendon force coordination problem has been extensively analysed in terms of the cost function, only few works have investigated the existence and sensitivity of solutions to fibre topology. The goal of this paper is to present an analysis of the solution set using the concepts of torque-feasible space (TFS) and wrench-feasible space (WFS) from cable-driven robotics. A shoulder model is presented and a simple musculo-tendon force coordination problem is defined. The ideal fibre model for representing muscles is reviewed and the TFS and WFS are defined, leading to the necessary and sufficient conditions for the existence of a solution. The shoulder model's TFS is analysed to explain the lack of anterior deltoid (DLTa) activity. Based on the analysis, a modification of the model's muscle fibre geometry is proposed. The performance with and without the modification is assessed by solving the musculo-tendon force coordination problem for quasi-static abduction in the scapular plane. After the proposed modification, the DLTa reaches $20 \%$ of activation.
\end{abstract}

Keywords: shoulder; musculoskeletal modelling; musculo-tendon force coordination; anterior deltoid; torquefeasible space

\section{Introduction}

The shoulder is essential in providing mobility for the upper limb. Dysfunctions limit concerned individuals in their daily activities. As such, a number of musculoskeletal models have been developed to improve our understanding of its physiology: the Delft shoulder and elbow model (DSEM) (van der Helm 1994a, 1994b; Nikooyan et al. 2010), the Swedish model (Högfors et al. 1987, 1991; Karlsson and Peterson 1992; Makhsous et al. 1999), the Visible Human Project (VHP) model (Garner and Pandy 1999, 2001; Quental et al. 2012), the shoulder model of the AnyBody modelling software (Damsgaard et al. 2006), the Stanford model available in opensim (Holzbaur et al. 2005; Webb 2011) and the Newcastle model (Charlton and Johnson 2006). There are a number of other models that have been reviewed in the literature (Yang et al. 2010; Prinold et al. 2013).

Many of the previously listed models are designed to solve the musculo-tendon force coordination or recruitment problem (Erdemir et al. 2007). Consisting in estimating the musculo-tendon and joint reaction forces associated with movements of the system, this problem is particularly challenging for shoulder models because of the musculature's geometric complexity. It is difficult to obtain solutions that reproduce in vivo measurements of muscle activity and joint loads.
In musculoskeletal shoulder models, the anterior deltoid's (DLTa) activity is frequently underestimated. In vivo measurements have shown the DLTa to contribute significantly to movements of the upper limb such as abduction (Escamilla et al. 2009). However, a number of models predict insufficient activity, including the VHP model (Quental et al. 2015), the DSEM model (van der Helm 1994a, 1994b; Quental et al. 2015), the AnyBody model (Sins 2014), the Newcastle model (Masjedi and Johnson 2010) and others (Dickerson et al. 2007; Yanagawa et al. 2008).

Model-estimated and measured muscle activity differ because of how skeletal muscles are modelled. A skeletal muscle is a large collection of fibres applying a distributed force to the skeletal system. Models use a discrete number of massless, frictionless fibres from origin to insertion to represent a muscle's line of action. This is called the ideal fibre model and there exist multiple algorithms for building the fibre's path from origin to insertion. There are straight line methods (Seireg and Arvikar 1973). There are static and dynamic via point methods (Delp et al. 1990; Carman and Milburn 2005). The path is defined by one or more straight lines passing through points that are fixed with respect to the bones or move to adjust the fibre's path. There are algorithms that construct a path representing the fibre passing through the centroids of the muscle's cross sections. The path wraps over simple geometric surfaces

*Corresponding author. Email: david.ingram@epfl.ch 
representing the underlying bony structures (Raikova 1992; Garner and Pandy 2000). More recent algorithms use optimisation with complex geometric shapes to impose a topology of the fibre's path (Audenaert and Audenaert 2008; Desailly et al. 2010). Finite element models are also being developed (Webb 2011). The use of the ideal fibre model raises two questions: how many cables are needed and how to define fibre path geometry from origin to insertion? These questions are directly related to the existence of a solution to the musculo-tendon force coordination problem (ValeroCuevas et al. 2007).

The coordination problem has been extensively studied with respect to the cost function (Erdemir et al. 2007), but the existence and sensitivity of solutions to fibre topology have been poorly investigated. The minimum number of ideal fibres needed to represent large muscles has been investigated using a rank condition on the moment-arm matrix (van der Helm and Veenbaas 1991). A model of a finger was used to determine the set of loads a musculoskeletal model can resist (Valero-Cuevas et al. 1998). An efficient method of determining if a model can resist a prescribed set of loads has also been developed (Chu and Hughes 2010). How to model the musculature using ideal fibres remains a challenging problem.

The goal of this paper is to present a method of analysing the use of the ideal cable to model in a musculoskeletal model of the shoulder, built using the muscle wrapping framework of the VHP model. The model considers the shoulder's kinematics and dynamics and includes 16 muscles represented by 28 ideal fibres representing the centroid fibres (Garner and Pandy 2000). Inverse-dynamics and static optimisation are used to coordinate the musculo-tendon forces. The ideal fibre model is reviewed to explain the link between the topology of a muscle's line of action and its actuation on the skeletal system. The concepts of wrench-feasible space (WFS) and torque-feasible space (TFS) are introduced to state the necessary and sufficient conditions for the existence of a solution. An analysis of the model's TFS is presented, yielding answers to the DLTa's limited activity in the model. An improved model of the musculature is proposed and shown to yield higher DLTa activation levels. This paper concludes with a discussion on the use of WFS and TFS to improve geometric muscle models.

\section{Methods}

\subsection{A musculoskeletal model of the human shoulder}

The model is constructed from MRI scans of an adult male's right shoulder, including bony landmarks, muscle origins, and insertions and muscle fibre paths. The model includes the thorax, clavicle, scapula and humerus. The arm is considered to be outstretched and the thorax is the

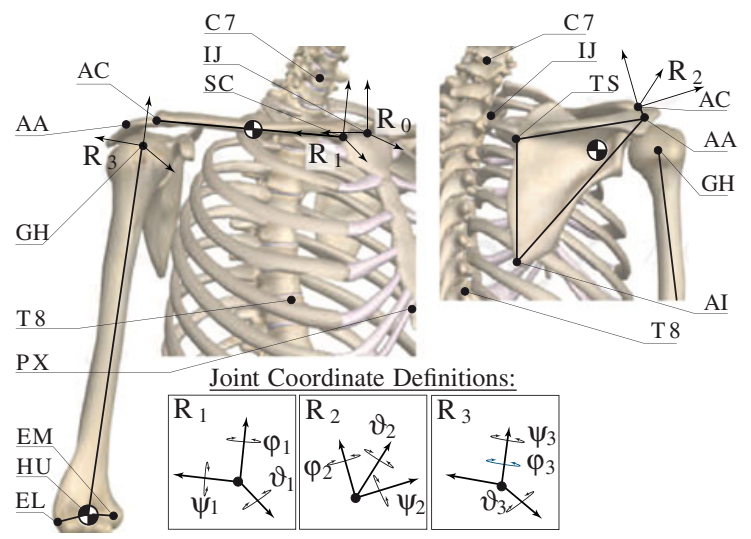

Figure 1. Illustration of the bony landmarks, reference frames and bone centres of gravity. Image created using ZygoteBody ${ }^{\mathrm{TM}}$ zygotebody.com.

carrier body. Reference frames are defined for the thorax $\mathcal{R}_{0}$ (inertial frame), clavicle $\mathcal{R}_{1}$, scapula $\mathcal{R}_{2}$ and humerus $\mathcal{R}_{3}$ (Figure 1). The subindexes identify each frame. The sternoclavicular (SC), acromioclavicular (AC) and glenohumeral (GH) articulations are modelled as ideal ball and socket joints (spherical kinematics pairs). The model's configuration is parameterised by a vector of nine kinematic coordinates, three angles per joint.

$$
\vec{\kappa}=\left(\begin{array}{lllllllll}
\psi_{1} & \vartheta_{1} & \varphi_{1} & \psi_{2} & \vartheta_{2} & \varphi_{2} & \psi_{3} & \vartheta_{3} & \varphi_{3}
\end{array}\right)^{T} .
$$

The reference frames and rotation angles are defined according to the ISB guidelines (Wu et al. 2005). The scapulo-thoracic contact is modelled by defining holonomic constraints between the two end-points on the scapula's medial border (TS and $A I$ ) and two ellipsoids approximating the contact surface (Ingram et al. 2013a, $2013 \mathrm{~b}$ ). The model has seven degrees of freedom $(D O F=7)$. There are nine kinematic coordinates subject to two holonomic constraints.

Each body is given a mass $M_{i}$ and an inertia $\mathcal{I}_{i}$ defined with respect to the centre of gravity (Klein Breteler et al. 1999). The humerus and arm are considered to be a single body with the centre of gravity at the elbow joint $H U$. The equations of motion are obtained using analytical mechanics (Hand and Finch 1998). The generalised coordinates are defined as the kinematic coordinates. The dynamic model is defined by

$$
\begin{gathered}
\frac{\partial^{2} \mathcal{L}}{\partial \dot{\vec{\kappa}}^{2}} \ddot{\vec{\kappa}}+\frac{\partial^{2} \mathcal{L}}{\partial \vec{\kappa} \partial \dot{\vec{\kappa}}} \dot{\vec{\kappa}}-\frac{\partial \mathcal{L}}{\partial \vec{\kappa}}=\vec{t}_{g}+\left(\frac{\partial \vec{\Phi}}{\partial \vec{\kappa}}\right)^{T} \vec{\lambda}, \\
\text { s.t. } \vec{\Phi}(\vec{\kappa})=\overrightarrow{0},
\end{gathered}
$$

where $\mathcal{L}$ is the sum of the bone lagrangians. The vector $\vec{t}_{g}$ is the vector of generalised external forces and $\vec{\lambda}$ is the vector of lagrangian multipliers associated with the 
scapulo-thoracic constraints $\Phi$, dealt with using the gradient projection method (Baumgarte 1983).

This study only considers the action of the muscles on the skeletal system. The musculature is modelled using the framework from the VHP model where muscles are represented by a discrete number of massless, frictionless fibres wrapping over the skeletal structure (Garner and Pandy 2000, 2001). The model includes 16 anatomical muscles represented by 28 ideal fibres that each parameterise a muscle's centroid fibre, passing through the centroids of the muscle's cross sections. The MRI data were used to build the entire model but the type of wrapping surfaces and the number of cables are the same as in the VHP model. The vector of generalised external forces $\vec{t}_{g}$ is defined by

$$
\vec{t}_{g}=\mathbf{P}_{0}(\vec{\kappa}) \vec{t}_{a}=\mathbf{P}_{0}(\vec{\kappa}) \mathbf{W}(\vec{\kappa}) \vec{f}=\mathbf{W}_{0}(\vec{\kappa}) \vec{f},
$$

where $\vec{f}$ is the vector of muscle force intensities. The vector $\vec{t}_{a}$ is the torques around the inertial frame axes centred at each of the three joints. The matrix $\mathbf{W}_{0}(\vec{\kappa})$ is the generalised moment-arm matrix resulting from the projection of the moment-arms $\mathbf{W}(\vec{\kappa})$ into the generalised coordinate space (Aeberhard et al. 2009; Ingram et al. 2013a, 2013b). The projection matrix $\mathbf{P}_{0}(\vec{\kappa})$ is defined as the partial derivative of the rotational velocity vectors with respect to the coordinate velocities (Aeberhard et al. 2009). The tendon excursion method computes the generalised moment-arms directly (Ingram et al. 2013a, 2013b). Muscles can only pull and have a maximum isometric strength $\vec{f}_{\max }$. The muscle force intensities vector is subject to the following constraints:

$$
\overrightarrow{0} \leq \vec{f} \leq \vec{f}_{\max }
$$

\subsection{Musculo-tendon force coordination}

The musculo-tendon force coordination or recruitment problem is defined in this paper as computing the musculo-tendon force intensities for a desired motion. Musculo-tendon force is defined as the overall force applied by the muscle on the bone. Motion is defined as a temporal evolution of the joint coordinates and their firstand second-order derivatives $(\vec{\kappa}(t), \dot{\vec{\kappa}}(t), \ddot{\vec{\kappa}}(t)), t \in\left[t_{0}, t_{f}\right]$. Given the desired motion, the dynamic model is inverted (2)-(3), yielding the required actuation torque at each joint. The muscle segment activations are found by inverting the torque-force map.

$$
\vec{t}_{g}(\vec{\kappa}, \dot{\vec{\kappa}}, \ddot{\vec{\kappa}})=\mathbf{W}_{0}(\vec{\kappa}) \vec{f} .
$$

The map is mathematically under-determined and there are an infinite number of solutions. A nonlinear program $(\mathrm{NLP})$ is defined at discrete instances $t_{k} \in\left[t_{0}, t_{f}\right]$ $(k=1,2, \ldots, n)$ to select a single solution. The cost function is defined as the sum of squared muscle stresses (van der Helm 1994a, 1994b; Rasmussen et al. 2001; Damsgaard et al. 2006). Muscle stress is defined as the muscle's force divided by the physiological crosssectional area. The problem is subject to the torqueforce relation (6), the physical limitations on the muscle forces (5) and to glenohumeral joint stability constraints. The stability constraints keep the orientation of the glenohumeral joint reaction force (GH-JRF) inside an ellipse, approximating the glenoid (van der Helm 1994a, 1994b; Lemieux et al. 2013) The GH-JRF $\vec{f}_{r}$ is computed from the expression of the arm's translational dynamics in the inertial frame (Newton's second law of motion).

$$
M_{3} \ddot{\vec{x}}_{0,3}=M_{3} \vec{g}_{0}+\mathbf{D}_{0} \vec{f}+\vec{f}_{r}
$$

where $M_{3}$ is the arm's mass. The vector $\ddot{\vec{x}}_{0,3}$ is the arms translational acceleration. The vector $\vec{g}_{0}$ is the earth's gravitational vector field. The matrix $\mathbf{D}_{0}$ is the force direction matrix. The static optimisation at instant $t_{k}$ is defined by

$$
\begin{gathered}
\min _{\vec{f}(t k)} \Gamma(\vec{f}(t k))=\frac{1}{2} \sum_{j=1}^{p} \frac{(\vec{f}(t k))_{j}^{2}}{P C S A_{j}^{2}}=\frac{1}{2} \vec{f}(t k)^{T} \mathbf{P} \vec{f}(t k), \\
(\mathbf{P})_{i, j}=\frac{\delta_{i, j}}{P C S A_{j}^{2}} \\
\text { s.t. } \vec{t}_{g}\left(\vec{k}\left(t_{k}\right), \vec{k}\left(t_{k}\right), \ddot{\vec{k}}\left(t_{k}\right)\right)=\mathrm{W}_{0}(\vec{k}(t k)) \vec{f}(t k), \\
\text { Torque }- \text { force constraint, } \\
\overrightarrow{0} \leq \vec{f} \leq \vec{f}_{\text {max }}, \quad \text { Min/max force bounds, }
\end{gathered}
$$

$$
\vec{g}\left(\vec{k}\left(t_{k}\right), \vec{k}\left(t_{k}\right), \vec{k}\left(t_{k}\right), \vec{f}\left(t_{k}\right)\right) \leq \overrightarrow{0}, \quad \text { GH stability constraint. }
$$

The NLP is solved using the null-space optimisation technique from Aeberhard et al. (2009); Terrier et al. (2010).

\subsection{Ideal muscle fibre model}

Skeletal muscles are built as a collection of parallel fibres. The fibres attach at either end to bones in the skeletal structure. The attachment sites are surfaces, covering an area of the bone. The ideal fibre model represents muscles using a discrete number of fibres, reducing the attachment sites to a discrete number of points. The model is built on the principle that muscles apply a system of forces on the bones (Poppen and Walker 1978). A point on which the ideal fibre model differs from reality is that real muscles apply a continuous distribution of forces on the bones while ideal fibres apply a discrete number of forces. 
To illustrate this point, consider a muscle applying a distributed force on a bone. The first step towards constructing a model is to approximate the distribution with a discrete system of $\hat{m} \gg 1$ forces. Each force in the system is applied at a point $\hat{P}_{j}$. A force $\hat{\vec{f}}_{j}$ applied at a point $\hat{P}_{j}$ defines a screw $\hat{\mathcal{F}}_{Q}$ at any other point $Q$ (Ball 1876).

$$
\hat{\mathcal{F}}_{Q}=\left(\hat{\vec{f}}_{j}, \hat{\vec{r}}_{j} \times \hat{\vec{f}}_{j}\right)=\left(\hat{\hat{\vec{f}}_{j}}, \hat{\overrightarrow{t_{j}}}\right)
$$

where $\hat{\vec{r}}_{j}$ is the vector from $Q$ to $\hat{P}_{j}$ and $\hat{\vec{t}}_{j}$ the moment of force created by $\hat{\vec{f}}_{j}$ at $\mathrm{Q}$. A couple of force is a screw with zero force.

$$
\mathcal{F}=(\overrightarrow{0}, \vec{t}) .
$$

The system of $\hat{m}$ forces applied by a muscle on a bone can be reduced to a single screw defined with respect to a point $Q$ on the bone (Poinsot 1848).

$$
\hat{\mathcal{F}}_{Q}=\left(\sum_{j=1}^{\hat{m}} \hat{\vec{f}}_{j}, \sum_{j=1}^{\hat{m}}\left(\hat{\vec{r}}_{j} \times \hat{\vec{f}}\right)\right)=\left(\hat{\vec{f}}_{Q}, \hat{\vec{t}}_{Q}\right) .
$$

This screw defines the muscle's ability to actuate the bone with respect to the point $Q$.

Musculoskeletal systems have many muscles, and representing each muscle with a large system of forces can lead to numerical instability of the coordination problem. The ideal fibre model uses a small number of fibres to represent each muscle. Ideal fibres apply $1 \leq$ $m \ll \hat{m}$ forces $\vec{f}_{i}$ on the bones at points $\mathbf{P}_{i}$. For the model to represent real muscles, the forces applied by the ideal fibres must generate a screw $\mathcal{F}_{Q}$ that is approximately equal to the screw $\hat{\mathcal{F}}_{Q}$ generated by the system of forces.

$$
\mathcal{F}_{Q}=\left(\sum_{i=1}^{m} \vec{f}_{i}, \sum_{i=1}^{m}\left(\vec{r}_{i} \times \vec{f}_{i}\right)\right)=\left(\vec{f}_{Q}, \vec{t}_{Q}\right) \approx \hat{\mathcal{F}}_{Q}=\left(\hat{\vec{f}}_{Q}, \hat{\vec{t}}_{Q}\right) .
$$

The ideal fibre model also makes the assumption that the force within each fibre is the same throughout the fibre. All the forces applied by an ideal fibre have the same magnitude $f$ and the screw $\mathcal{F}_{Q}$ can be expressed by

$$
\begin{aligned}
\mathcal{F}_{Q} & =\left(\sum_{i=1}^{m} \vec{f}_{i}, \sum_{i=1}^{m}\left(\vec{r}_{i} \times \vec{f}_{i}\right)\right) \\
& =f \cdot\left(\sum_{i=1}^{m} \vec{d}_{i}, \sum_{i=1}^{m}\left(\vec{r}_{i} \times \vec{d}_{i}\right)\right)=f \cdot(\vec{d}, \vec{w}),
\end{aligned}
$$

where $\vec{d}$ is the resulting force direction vector and $\vec{w}$ is the resulting moment-arm at $Q$. The ideal fibre model represents the muscle's ability to actuate the bone using two vectors that form a wrench of magnitude $f$ : a force direction vector $\vec{d}$ and a moment-arm $\vec{w}$. The vector $\vec{w}$ is not a generalised moment-arm but a moment-arm around orthogonal axes centred at $Q$. The ideal fibre model should always be analysed in terms of this wrench. Furthermore, the wrench is dependent on the choice of $Q$, a point that is essential. The same wrench can be expressed by another wrench with respect to a different point $Q^{\prime}$ minus a couple of force (Poinsot 1848).

$$
\begin{aligned}
\mathcal{F}_{Q}= & \mathcal{F}_{Q^{\prime}}-(0, \vec{t})=f\left(\sum_{i=1}^{m} \vec{d}_{i}, \sum_{i=1}^{m}\left(\vec{y}_{i} \times \vec{d}_{i}\right)\right) \\
& -f\left(\overrightarrow{0}, \sum_{i=1}^{m}\left(\left(\vec{y}_{i}-\vec{r}_{i}\right) \times \vec{d}_{i}\right)\right) .
\end{aligned}
$$

The couple of force between the two screws can be substantial, depending on how distant $Q$ is from $Q^{\prime}$. When analysing a muscle's ability to actuate a bone, the point $Q$ should be chosen appropriately. In musculoskeletal models, the skeletal dynamics are defined in terms of joint coordinates. Also, the tendon excursion method computes generalised moment-arms with respect to joint centres and their coordinates. Therefore, the ability of the ideal fibre model to represent muscles should always be assessed in terms of the muscle's ability to actuate bones with respect to the joints. Often, the number ideal fibres and their topology are selected arbitrarily, leading to considerable differences between $\mathcal{F}_{Q}$ and $\hat{\mathcal{F}}_{Q}$. Furthermore, ideal fibre path construction algorithms, like the centroid line approach, focus on the geometric fidelity of the path (Desailly et al. 2010) and not on the fibre's mechanical fidelity in terms of the wrench $\mathcal{F}_{Q}$.

The ideal fibre model should be built using the wrench $\mathcal{F}_{Q}$ and $Q$ should be a joint centre because the resulting wrench is used to construct the torque-force map and governs the solutions of the musculo-tendon force coordination problem. The following sections analyse the effect of muscles on the joints.

\subsection{Existence of a solution: necessary and sufficient conditions}

In the NLP defined in Section 2.2, the cost function provides a means of differentiating the solutions within the solution space. The solution space is defined by the constraints and the space of decision variables. In this context, the decision variables are the forces within the ideal muscle fibres and the solution space is defined by the torque-force map (9). The physical bounds (10) and GH joint stability constraint (11) further restrict the solution space to a subspace. The remainder of this section discusses the solution space, defined only in terms of the torque-force map and physical bounds because they are the two constraints found in almost every musculo-tendon force coordination problem.

The inverse dynamics model defines a map between the joint kinematics space $\mathscr{K}_{S} \subset \mathbb{R}^{9}$ and the actuation 
space $\mathscr{T}_{S} \subset \mathbb{R}^{9}$ (Nof 1999).

$$
\begin{gathered}
\mathfrak{D}^{-1}: \mathscr{K}_{S} \rightarrow \mathscr{T}_{S}, \\
(\vec{\kappa}, \dot{\vec{\kappa}}, \ddot{\vec{\kappa}}) \mapsto \mathfrak{D}(\vec{\kappa}, \dot{\vec{\kappa}}, \ddot{\vec{\kappa}})=\vec{t}_{g}(\vec{\kappa}, \dot{\vec{\kappa}}, \ddot{\vec{\kappa}}) .
\end{gathered}
$$

The dynamic model can always be inverted. Exceptions include singular points due to the choice of coordinates. Computing the required actuation is therefore straightforward once $\mathfrak{D}^{-1}$ is constructed. The challenge lies in inverting the torque-force map, and the existence of a solution is intrinsically linked to this inversion.

The torque-force map governs the coordination problem, defining a map between the muscle force intensities space $\mathscr{F}_{S} \subset \mathbb{R}^{28}$ and the actuation space.

$$
\begin{gathered}
\mathfrak{M}_{\vec{\kappa}}: \mathscr{F}_{S} \rightarrow \mathscr{T}_{S}, \\
\vec{f} \mapsto \mathfrak{M}_{\vec{\kappa}}(\vec{f})=\mathbf{W}_{0}(\vec{\kappa}) \vec{f}=\mathbf{P}_{0}(\vec{\kappa}) \mathbf{W}(\vec{\kappa}) \vec{f}=\mathbf{P}_{0}(\vec{\kappa}) \vec{t}_{a}=\vec{t}_{g}(\vec{\kappa}, \vec{f}) .
\end{gathered}
$$

In a given configuration $\vec{\kappa}$, the torque-force map defines a linear map between two vector spaces. The map's domain is the force intensity space $\mathscr{F}_{S} \subset \mathbb{R}^{28}$. The map's image space is the actuation space $\mathscr{T}_{S} \subset \mathbb{R}^{9}$. The map is dependent on the configuration $\vec{\kappa}$. For a system with 28 ideal muscle fibres, the upper and lower bounds (10) on the forces (without additional constraints) define the map's domain space $\mathscr{F}_{S}$ as a convex polytope ${ }^{1}$ in $\mathbb{R}^{28}$. The geometry of the domain space polytope is defined by the values of $\vec{f}_{\max }$. The geometry of the image space polytope is defined by the generalised moment-arms matrix $\mathrm{W}_{0}(\vec{\kappa})$ which changes with the configuration. The image of a convex polytope under a linear transformation is also a convex polytope (Grunbaum 2003). The image space of the torque-force map under the physical bounds is therefore also a convex polytope: $\mathfrak{M}_{\vec{\kappa}}\left(\mathscr{F}_{S}\right) \subset \mathscr{T}_{S}$ and is called the TFS (Figure 2).

Definition 2.1. TFS $\mathscr{T}_{f s}$ set of actuation vectors that the muscle fibres are able to produce under the physical bounds

$$
\mathscr{T}_{f s}=\mathfrak{M}_{\widetilde{\kappa}}\left(\mathscr{F}_{S}\right) \subset \mathscr{T}_{s} .
$$

Associated with the definition of TFS is the definition of WFS. The WFS is all kinematic configurations mapped by $\mathfrak{D}^{-1}$ to actuation vectors inside the TFS. The WFS is not a convex polytope.

Definition 2.2. WFS $\mathscr{W}_{f s}$ set of kinematic configurations

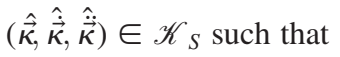

$$
\mathfrak{D}^{-1}(\hat{\vec{\kappa}}, \hat{\vec{\kappa}}, \hat{\overrightarrow{\tilde{\kappa}}}) \in \mathscr{T}_{f s} .
$$

If the desired kinematics remain within the wrenchfeasible space, the required actuation vector is inside the TFS which is the image space of the torque-force map. If the moment-arm matrix has full column rank, the torqueforce map can be inverted and there exists a solution (van der Helm and Veenbaas 1991; Gouttefarde et al. 2007). This analysis allows one to state the necessary and sufficient conditions for the existence of a solution to the musculo-tendon force coordination problem.

Theorem 2.3. At any discrete instance $t_{k}$, a solution to the musculo-tendon force coordination problem exists if and only if $\left(\hat{\vec{\kappa}}\left(t_{k}\right), \hat{\vec{\kappa}}\left(t_{k}\right), \hat{\vec{\kappa}}\left(t_{k}\right)\right) \in \mathscr{W}_{f s}$ and the generalised moment-arm matrix $\mathbf{W}_{0}\left(\vec{\kappa}\left(t_{k}\right)\right)$ has full column rank.

Guaranteeing the conditions of the previous theorem is challenging. The rank condition on the moment-arm matrix is fairly straightforward, but determining the WFS is not. This is especially true for the shoulder, given the geometric complexity of its musculature.

\subsection{Torque feasibility of the shoulder}

The musculoskeletal shoulder model described in Section 2.1 and the VHP model (Garner and Pandy 2000, 2001) share the same muscle fibre topology (number of fibres and types of wrapping surfaces) but different numerical anatomic data-sets. When the model was initially tested, the estimated DLTa activity was zero. This section presents an analysis of the model's TFS that explains why the DLTa's activity is underestimated.

Both $\mathfrak{D}^{-1}$ and $\mathfrak{M}_{\vec{K}}$ map into the actuation space, therefore, the geometry of the TFS contains a significant amount of information regarding the solutions to the musculo-tendon force coordination problem. The geometry of the TFS for a configuration $\vec{\kappa}$ is defined by either the generalised moment-arm matrix $\mathbf{W}_{0}(\vec{\kappa})$ or the moment-arms matrix $\mathbf{W}(\vec{\kappa})$. The first yields the TFS in the generalised coordinate space, the second yields the TFS in the absolute inertial reference frame $\left(\mathcal{R}_{0}\right)$. One can switch between the two using the projection matrix $\mathbf{P}_{0}(\vec{\kappa})$. Using the moment-arm matrix, the TFS for the shoulder model was computed for its resting configuration (arm down). The TFS was computed in the inertial frame because it has an easier interpretation in terms of torques around orthogonal axes in space. The computation was

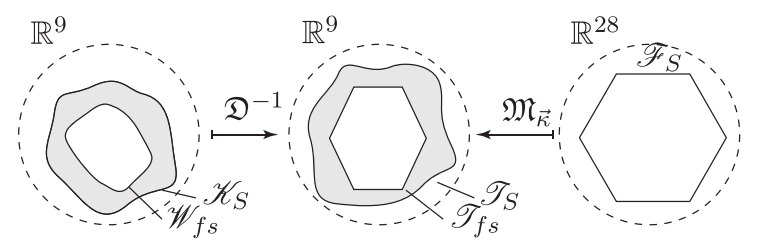

Figure 2. Illustration of the relation between the joint kinematic space $\mathscr{K}_{S}$, actuation space $\mathscr{T}_{S}$ and muscle force intensities space $\mathscr{F}_{S}$. The torque and TFSs are also shown as well as the wrench and wrench-feasible sets. 
done using 28 ideal muscle fibres. The model has three spherical joints and the TFS can be separated into three parts. One for each joint and each part is a convex polytope in $\mathbb{R}^{3}$, defined with respect to $\mathcal{R}_{0}$ but centred at a joint. A visualisation of these polytopes shows the AC and GH TFSs (GH-TFS, AC-TFS) to be threedimensional volumes but not the SC TFS (SC-TFS) (Figure 3). The SC-TFS is visually almost twodimensional.

The torque-force map between muscle forces and the torques in the inertial frame is decomposed into three parts relating muscle force intensities to the torques they create around the joints.

$$
\mathbf{P}_{0}^{-1}(\vec{\kappa}) \vec{t}_{g}=\vec{t}_{a}=\left(\begin{array}{c}
\vec{t}_{a, S C} \\
\vec{t}_{a, A C} \\
\vec{t}_{a, G H}
\end{array}\right)=\mathbf{W}(\vec{\kappa}) \vec{f}=\left(\begin{array}{c}
\mathbf{W}_{S C} \\
\mathbf{W}_{A C} \\
\mathbf{W}_{G H}
\end{array}\right) \vec{f} .
$$

The part of this equation related to the $\mathrm{SC}$ joint is projected into the clavicle's reference frame $\mathcal{R}_{1}$, using the rotation matrix $\boldsymbol{R}_{0,1}$.
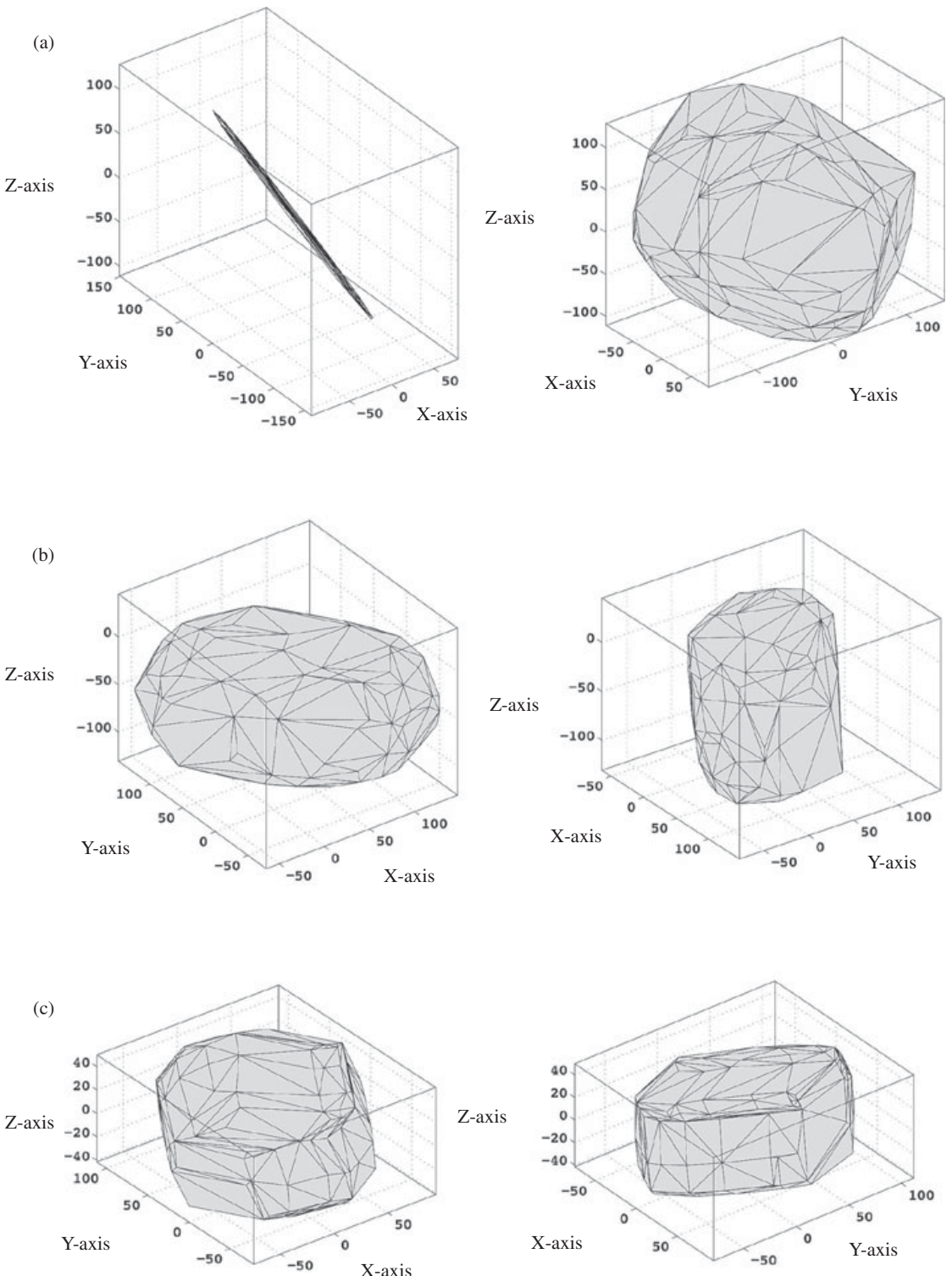

Figure 3. Visualisation of the sternoclavicular (a), acromioclavicular (b) and glenohumeral (c) TFSs. The spaces are computed for the shoulder model's resting position and visualised in two spatial orientations. 


$$
\boldsymbol{R}_{0,1} \vec{t}_{a, S C}=\left(\begin{array}{c}
t_{a, x} \\
t_{a, y} \\
t_{a, z}
\end{array}\right)=\boldsymbol{R}_{0,1} \boldsymbol{W}_{S C} \vec{f}=\boldsymbol{W}_{p, S C} \vec{f}+\boldsymbol{W}_{n, S C} \vec{f}
$$

The projected moment-arm matrix is decomposed into a matrix containing the moment-arm components parallel to the SC-AC axis (z-axis, subindex $p$ ) and a matrix containing the normal terms ( $x$ and $y$ axes, subindex $n$ ). The projected moment-arm matrix has a line with only four non-zero elements.

where

$$
\mathbf{R}_{0,1} \mathbf{W}_{S C}=\mathbf{W}_{p, S C}+\mathbf{W}_{n, S C},
$$

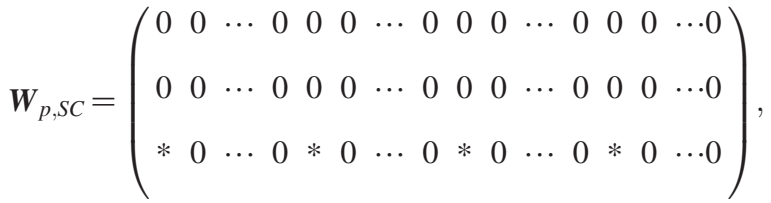

$$
\begin{aligned}
& \boldsymbol{W}_{n, S C}=\left(\begin{array}{ccc}
* & \cdots & * \\
* & \cdots & * \\
0 & \cdots & 0
\end{array}\right) \text {. }
\end{aligned}
$$

In the moment-arm matrix, each column contains the moment-arms of one of the ideal muscle fibres. Each line corresponds to one of the joints axes. The four non-zero elements in the projected moment-arm matrix are associated with four muscle fibres (marked by a (*) in Table 1): the subclavius (SBCL), the superior part of the trapezius (TRPs), the superior part of the pectoralis major (PMJs) and the DLTa. The line containing the four nonzero elements is the SC joint axis parallel to the SC-AC axis. Only four muscles contribute to the clavicle's rotation around its longitudinal axis. This is expressed by the equation

$$
\begin{aligned}
t_{a, z}= & w_{z, \mathrm{SBCL}} f_{\mathrm{SBCL}}+w_{z, \mathrm{TRPs}} f_{\mathrm{TRPs}}+w_{z, \mathrm{PMJs}} f_{\mathrm{PMJs}} \\
& +w_{z, \mathrm{DLTa}} f_{\mathrm{DLTa}} .
\end{aligned}
$$

This result is also explained by the following reasoning. The SC-TFS is defined by the first 20 ideal muscle fibres (Table 1). There are four ideal muscle fibres originating or inserting directly on the clavicle (marked by a star in Table 1). The remaining 16 ideal muscle fibres originate on the thorax and insert on the scapula. These ideal muscle fibres do not attach to the clavicle that has the following

\begin{tabular}{|c|c|c|c|c|c|}
\hline Muscle name & Origin & Insertion & SC & $\mathrm{AC}$ & GH \\
\hline (*)Subclavius (SBCL) & Sternum & Clavicle & $\vec{w}_{1,1}$ & $\overrightarrow{0}$ & $\overrightarrow{0}$ \\
\hline Serratus anterior 1 (SRAs) & Thorax & Scapula & $\vec{w}_{1,2}$ & $\vec{w}_{2,2}$ & $\underline{0}$ \\
\hline Serratus anterior 2 (SRAm) & Thorax & Scapula & $\vec{w}_{1,3}$ & $\vec{w}_{2,3}$ & $\overrightarrow{0}$ \\
\hline Serratus anterior 3 (SRAi) & Thorax & Scapula & $\vec{w}_{1,4}$ & $\vec{w}_{2,4}$ & $\underline{0}$ \\
\hline (*)Trapezius 1 (TRPs) & Thorax & Clavicle & $\vec{w}_{1,5}$ & $\overrightarrow{0}$ & $\overrightarrow{0}$ \\
\hline Trapezius 2 (TRPms) & Thorax & Scapula & $\vec{w}_{1,6}$ & $\vec{w}_{2,6}$ & $\underline{0}$ \\
\hline Trapezius 3 (TRPmi) & Thorax & Scapula & $\vec{w}_{1,7}$ & $\vec{w}_{2,7}$ & $\overrightarrow{0}$ \\
\hline Trapezius 4 (TRPi) & Thorax & Scapula & $\vec{w}_{1,8}$ & $\vec{w}_{2,8}$ & $\underline{0}$ \\
\hline Levator scapulae (LVS) & Thorax & Scapula & $\vec{w}_{1,9}$ & $\vec{w}_{2,9}$ & $\overrightarrow{0}$ \\
\hline Rhomboid minor (RMN) & Thorax & Scapula & $\vec{w}_{1,10}$ & $\vec{w}_{2,10}$ & 0 \\
\hline Rhomboid major 1 (RMJs) & Thorax & Scapula & $\vec{w}_{1,11}$ & $\vec{w}_{2,11}$ & $\overrightarrow{0}$ \\
\hline Rhomboid major 2 (RMJi) & Thorax & Scapula & $\vec{w}_{1,12}$ & $\vec{w}_{2,12}$ & 0 \\
\hline Pectoralis minor (PMN) & Thorax & Scapula & $\vec{w}_{1,13}$ & $\vec{w}_{2,8}$ & $\overrightarrow{0}$ \\
\hline (*)Pectoralis major 1 (PMJs) & Clavicle & Humerus & $\vec{w}_{1,14}$ & $\vec{w}_{2,14}$ & $\vec{w}_{3,14}$ \\
\hline Pectoralis major 2 (PMJm) & Thorax & Humerus & $\vec{w}_{1,15}$ & $\vec{w}_{2,15}$ & $\vec{w}_{3,15}$ \\
\hline Pectoralis major 3 (PMJi) & Thorax & Humerus & $\vec{w}_{1,16}$ & $\vec{w}_{2,16}$ & $\vec{w}_{3,16}$ \\
\hline Latissimus dorsi 1 (LTDi) & Thorax & Humerus & $\vec{w}_{1,17}$ & $\vec{w}_{2,17}$ & $\vec{w}_{3,17}$ \\
\hline Latissimus dorsi 2 (LTDm) & Thorax & Humerus & $\vec{w}_{1,18}$ & $\vec{w}_{2,18}$ & $\vec{w}_{3,18}$ \\
\hline Latissimus dorsi 3 (LTDs) & Thorax & Humerus & $\vec{w}_{1,19}$ & $\vec{w}_{2,19}$ & $\vec{w}_{3,19}$ \\
\hline (*)DLTa & Clavicle & Humerus & $\vec{w}_{1,20}$ & $\vec{w}_{2,20}$ & $\vec{w}_{3,20}$ \\
\hline Middle Deltoid (DLTm) & Scapula & Humerus & $\overrightarrow{0}$ & $\vec{w}_{2,21}$ & $\vec{w}_{3,21}$ \\
\hline Posterior Deltoid (DLTp) & Scapula & Humerus & 0 & $\vec{w}_{2,22}$ & $\vec{w}_{3,22}$ \\
\hline Supraspinatus (SUPR) & Scapula & Humerus & $\underline{0}$ & $\vec{w}_{2,23}$ & $\vec{w}_{3,23}$ \\
\hline Infraspinatus (INFR) & Scapula & Humerus & $\underline{0}$ & $\vec{w}_{2,24}$ & $\vec{w}_{3,24}$ \\
\hline Subscapularis (SBSC) & Scapula & Humerus & $\underline{0}$ & $\vec{w}_{2,25}$ & $\vec{w}_{3,25}$ \\
\hline Teres minor (TMN) & Scapula & Humerus & $\underline{0}$ & $\vec{w}_{2,26}$ & $\vec{w}_{3,26}$ \\
\hline Teres major (TMJ) & Scapula & Humerus & 0 & $\vec{w}_{2,27}$ & $\vec{w}_{3,27}$ \\
\hline Coracobrachialis (CRCB) & Scapula & Humerus & 0 & $\vec{w}_{2,28}$ & $\vec{w}_{3,28}$ \\
\hline
\end{tabular}
consequence: the 16 ideal muscle fibres not attached to the clavicle define moments of force around the SC joint

Table 1. Muscle segment moment-arms. This table describes where the muscle fibres define moment-arms given their origin and insertion 
through the force they transmit across the AC joint. These muscles apply a force on the scapula that is transmitted across the AC joint. Therefore, the moment-arm of the transmitted force is always perpendicular to the vector between the two joints. Out of the 20 ideal muscle fibres creating a moment-arm around the SC joint, 16 muscle fibres create moment-arms that are co-planar. The plane in which they lie is normal to the SC-AC axis.

Given the previous analysis, the underestimated DLTa's muscle activity can now be adequately explained. Expression (26) relates four muscle activities to the clavicle's longitudinal axis torque. In the musculo-tendon force coordination problem, $t_{a, z}$ is imposed through $\mathfrak{D}^{-1}$ and expression (26) is subject to the physical bounds. Therefore, the DLTa's predicted activation is either zero or very small with respect to its strength because muscle activations for the other three muscles cannot be found without violating (5).

\subsection{Improved muscle model}

In the present model, as in the VHP model, the TRPs and DLTa are represented by one segment each. In the real shoulder, the two muscles are antagonistic to each other. However, the moment-arms of both muscles $\left(w_{z}, \mathrm{TRPs}, w_{z, \mathrm{DLTa}}\right)$ around the clavicle's longitudinal axis have the same sign when the shoulder model is in the resting configuration (Figure 4). Therefore, the TRPs and DLTa are not modelled as antagonistic using the centroid line approach from the VHP framework. An implementation of the VHP model confirms this statement. The model built using the VHP wrapping framework, without modification, will subsequently be referred to as the 28 -fibre model.

The proposed modifications are defined to ensure that the TRPs and the DLTa are antagonistic. Antagonism is defined as having oppositely directed moment-arms. The moment-arms of either muscle, around the clavicle's longitudinal axis must have opposite signs.

$$
w_{z, \mathrm{TRPs}}, w_{z, \mathrm{DLTa}}<0 .
$$

This condition is satisfied if the TRPs muscle fibre insertion point is displaced anteriorly in the direction of the DLTa's origin surface (Figure 5).

The model is further improved if multiple fibres are used to represent the TRPs and DLTa. Indeed, it is easier to satisfy the equilibrium Equation (26) using more fibres. The improved model thus uses two fibres to represent the TRPs and DLTa. To define the fibres, the origin and insertion surfaces of both muscles are parameterised using third-order splines. The splines use three anchor points. One at either extremity of the attachment surface and one at the centroid point. These splines pass through the centroid point of the surfaces (Figure 5). For the TRPs, the centroid spline is displaced anteriorly in the direction of the DLTa's origin surface. The new fibres for the TRPs and DLTa are evenly distributed along the splines. The modified model adds two additional muscle fibres and will be referred to as the 30-fibre model.

\subsection{Model output}

The model has been implemented into the Mathworks computing environment using MRI scans of an adult male. The inertial data necessary to construct the dynamic model were taken from the literature (Klein Breteler et al. 1999). The types of wrapping surfaces needed to define the muscle fibres and the maximum isometric strength values were taken from the VHP model (Garner and Pandy 2001).

To evaluate the improvement of the 30-fibre model over the 28-fibre model, the muscle forces were computed

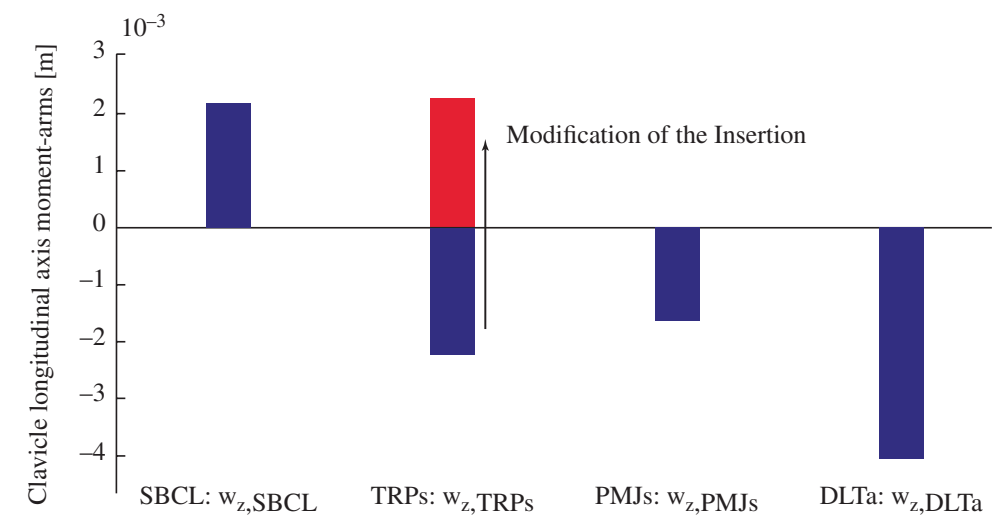

Figure 4. Moment-arms around the clavicle's longitudinal axis in the resting configuration for the SBCL, superior TRPs, PMJs and DLTa. 


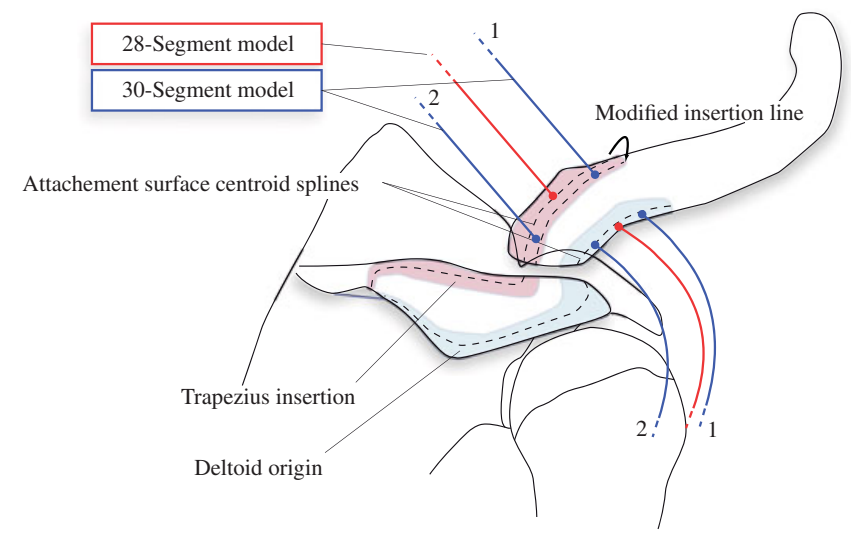

Figure 5. Illustration of the modifications brought to the shoulder model's muscle geometry. The superior TRPs fibre insertion points are displaced towards the DLTa's origin surface.

for quasi-static abduction in the scapular plane. The amount of torque $t_{a, z}(26)$ that can be compensated for by both models during the motion was also computed. This is the projection of the SC-TFS onto the $z$-axis, parallel to the $\mathrm{SC}-\mathrm{AC}$ axis. The performance is evaluated with respect to two points: muscle forces and GH-JRFs. The muscle forces are presented for quasi-static $(T=20 \mathrm{~s})$ abduction in the scapular plane. The kinematics of this motion were constructed using the methods from Ingram et al. (2013a, 2013b), El Habachi et al. (2014). The computation was done for 100 points evenly distributed between $0^{\circ}$ and $140^{\circ}$ abduction. The computation was stopped at $140^{\circ}$ because the model's validity is questionable for high abduction angles (Sins 2014). The muscle wrappings no longer faithfully represent the muscles. The musculo-tendon force coordination problem was performed for both the 28fibre and 30-fibre models described in the previous section. The joint reaction force intensity for quasi-static abduction is compared to results reported in van der Helm (1994a, 1994b), Bergmann et al. (2007), Terrier et al. (2008), Favre et al. (2009), Sins (2014). The intersection pattern between the joint reaction force and a planar approximation of the glenoid articular surface is compared to results reported in van der Helm (1994a, 1994b), Engelhardt et al. (2012).

\section{Results}

The improvement in terms of the torque $t_{a, z}$ that the muscles can compensate for is considerable (Figure 6). The 28-fibre model can generate very little positive torque $(\sim 0.3 \mathrm{Nm})$ around the clavicle's longitudinal axis and the amount of negative torque decreases as the arm abducts. The 30-fibre model can generate both positive and negative torque during the entire motion.

The model-predicted muscle forces are presented in percentage of the maximum isometric muscle force
(Figure 7). The muscle forces are presented for all the muscle fibres spanning the $\mathrm{GH}$ joint, not including the LTD and PMJ muscles (see Table 1 for abbreviations).

For the 28-fibre model, all the muscles apart from the DLTa are active during part or all the motion. The DLTa shows no activity. The DLTm, TMN and Coracobrachialis (CRCB) are the muscles showing the most activity. The TMN shows the highest activation level with 50\% activation at $100^{\circ}$ abduction. The DLTm, TMN and TMJ are the only muscles to remain active throughout the motion. All active muscles except the middle deltoid show an increase followed by a decrease in muscle force. The curves are all more or less bell shaped. In contrast, the middle deltoid shows a steady increase in muscle force throughout the entire motion.

For the 30-fibre model, the estimated muscle forces are very similar to the 28 -fibre model. However, the two DLTa

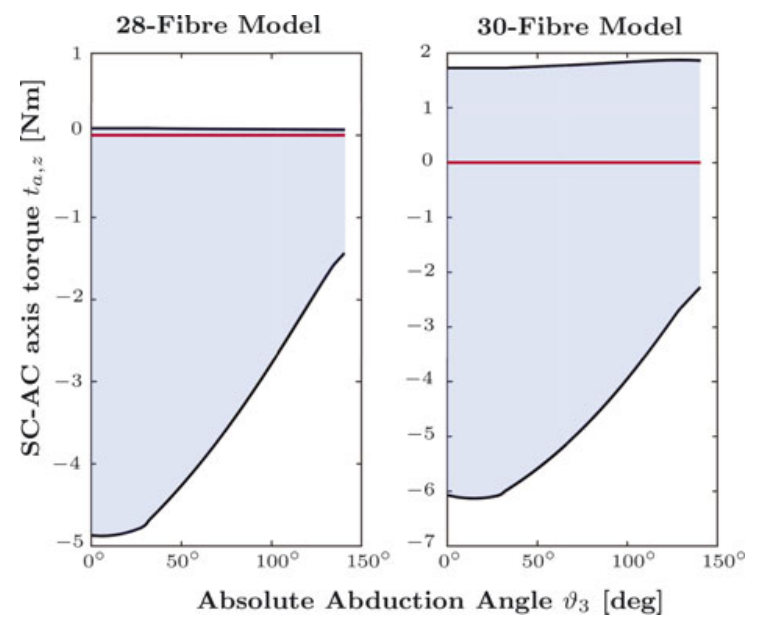

Figure 6. Torque $t_{a, z}$ (Equation (26)) around the clavicle's longitudinal axis that can be compensated for during quasi-static abduction in the scapular plane. 

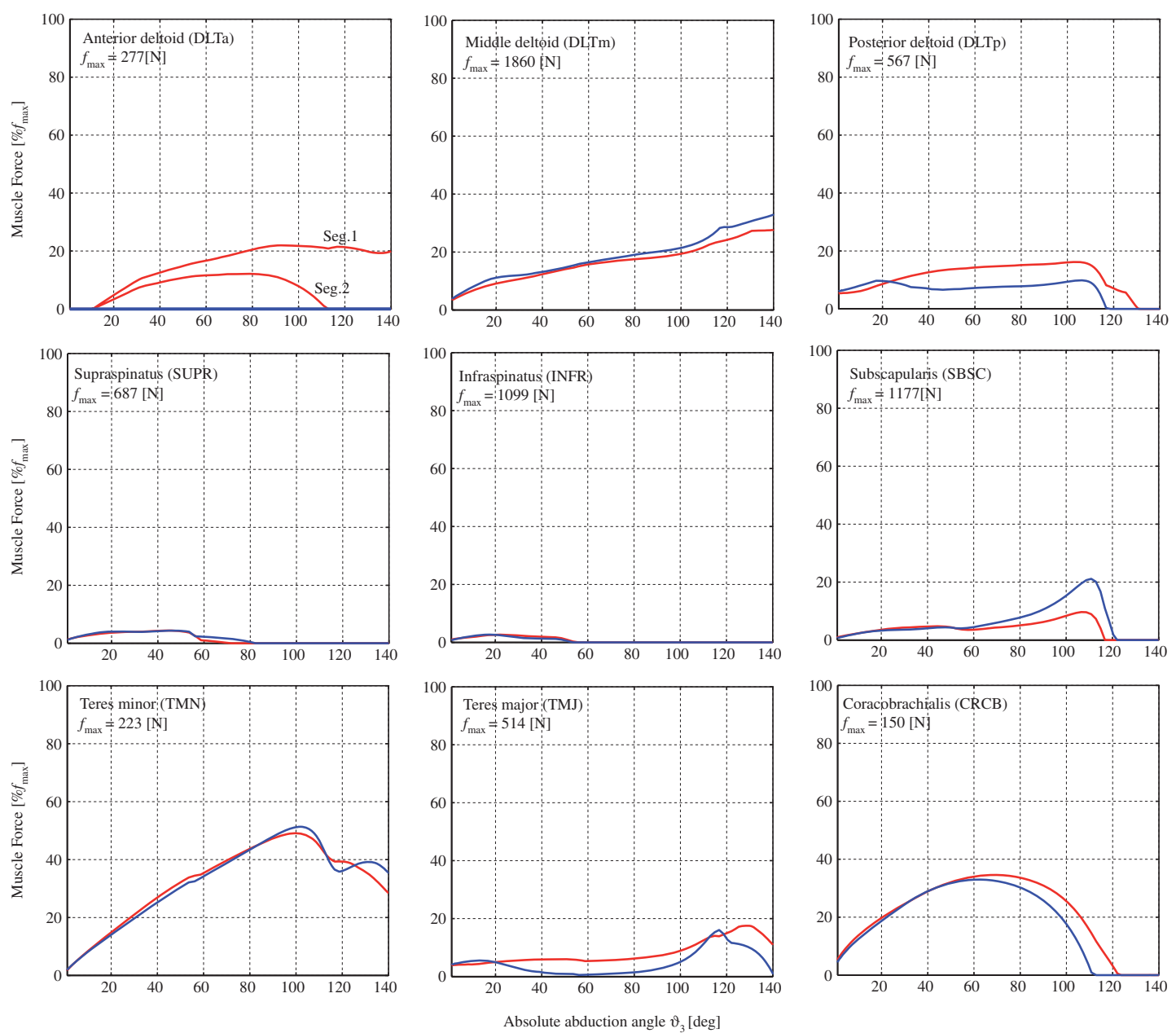

Figure 7. Comparison of estimated muscle forces during quasi-static abduction for the 28 -fibre model (blue line) and the 30 -fibre model (red line).

fibres show activity with the first segment reaching above $20 \%$ activation. The 30 -fibre model shows slightly more posterior deltoid activity and less subscapularis activity.

The GH-JRF is presented in terms of the force intensity in Newtons (Figure 8). The intersection point of the reaction force vector with the glenoid articular surface is also presented (Figure 9). For the 28-fibre model, during quasi-static abduction, the force intensity rises steadily from $140 \mathrm{~N}$ at $0^{\circ}$ abduction to a peak value of $880 \mathrm{~N}$ at $110^{\circ}$ abduction. Between $110^{\circ}$ and $140^{\circ}$ abduction, the force intensity slightly drops. The initial behaviour is consistent with other behaviours reported in the literature. The joint force direction is initially on the superioranterior edge (quadrant I) of the glenoid (Figure 9). From $0^{\circ}$ to $45^{\circ}$ abduction, the force initially moves posteriorly and then moves anteriorly between $0^{\circ}$ and $45^{\circ}$ abduction. After $45^{\circ}$ abduction the force moves downwards almost vertically. At $90^{\circ}$ abduction, the force is close to the middle axes in the anterior region (quadrant I). The force then continues to drop until $140^{\circ}$ abduction. The predicted behaviour is most similar to the behaviour from van der

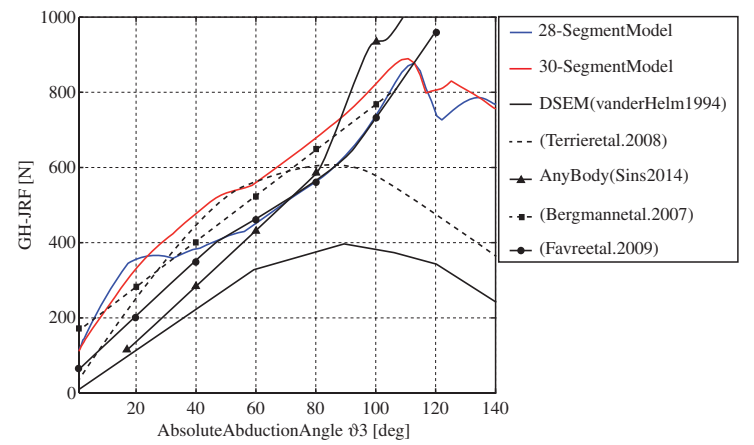

Figure 8. Comparison of GH-JRFs during quasi-static abduction in the scapular plane. 


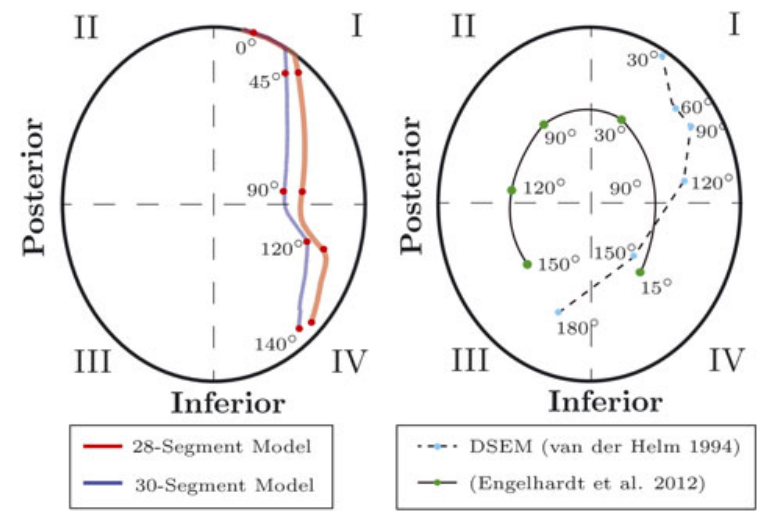

Figure 9. Intersection between the GH-JRFs and the glenoid articular surface. Comparison of model-predicted intersection patterns for quasi-static abduction in the scapular plane.

Helm (1994a, 1994b). The reaction force intensity and intersection pattern of the 30-fibre model differ slightly from those of the 28-fibre model. The 30-fibre force intensity increases faster and has a higher maximum value of $900 \mathrm{~N}$ at $110^{\circ}$ abduction. The 30-fibre intersection pattern is very similar to the 28 -fibre intersection pattern but slightly translated anteriorly.

\section{Discussion}

There are several musculoskeletal shoulder models in the literature (Karlsson and Peterson 1992; van der Helm 1994a, 1994b; Garner and Pandy 2001; Holzbaur et al. 2005; Damsgaard et al. 2006; Quental et al. 2012), developed for solving the musculo-tendon force coordination problem. However, some models present inconsistencies with in vivo measured data. An example is the model-estimated DLTa activity. Most models underestimate the DLTa's activity, including the DSEM (van der Helm 1994a, 1994b), VHP (Quental et al. 2015) and AnyBody (Sins 2014) models. Therefore, the goal of this paper was to present an analysis of the solution space of the musculo-tendon force coordination problem to determine the cause of DLTa's underestimated activity. The analysis was done using a shoulder model (28-fibre model) topologically almost identical to the VHP model from the literature (Garner and Pandy 2001; Quental et al. 2012). The model used in this paper was built using a different data-set collected from MRI scans but has the same number of bony landmarks and muscle fibres as the VHP model. Both models also use the same wrapping surfaces. The results of the analysis were used to improve the model (30-fibre model), the performance of which was assessed by solving the musculo-tendon force coordination problem.

The analysis introduced the definitions of TFS and WFS, leading to the necessary and sufficient conditions for the existence of a solution. It was also shown that the TFS provides key information regarding the solution itself including an explanation of the DLTa's inactivity in the model. The concepts of WFS and TFS have been used in cable-driven robots (Gouttefarde et al. 2007) and neuromuscular control (Sternad 2008), but have not till now been applied to the problem of musculo-tendon force coordination in musculoskeletal modelling.

The shoulder model presented in this paper is based on the VHP model and estimates similar muscle activation patterns. The 28-fibre model and the VHP model both predict no DLTa activation during all or part of quasistatic humeral abduction in the scapular plane. In contrast, the 30 -fibre model uses two additional cables resulting in $20 \%$ activation of the DLTa. Other than this significant difference in DLTa activity, the 28- and 30-fibre models perform very similarly. Both models predict GH-JRFs that agree well with model-estimated and in vivo measured data from the literature (Bergmann et al. 2007; Favre et al. 2009; Sins 2014). Both models also predict glenohumeral intersection patterns that are similar to the intersection pattern initially reported by the DSEM model (van der Helm 1994a, 1994b). Thus, the proposed modification to use two ideal fibres for the superior part of the TRPs and DLTa, instead of one, does not significantly change the overall solution, but does have a profound effect on the DLTa's activity. This leads to a remark concerning muscle modelling in general. At present, the theory of modelling muscles using fibres has not been fully developed.

There are a small number of results concerning the best way to represent muscles using ideal fibres. There is a rank criterion on the moment-arm matrix to determine the minimum number of fibres to accurately and efficiently represent the mechanical effect of muscles (van der Helm and Veenbaas 1991). There are algorithms to determine all or part of the loads a model can bear (Valero-Cuevas et al. 1998; Chu and Hughes 2010). However, the number of cables and their topology are often selected arbitrarily leading to considerable differences between model estimated and in vivo measured muscle activities. To illustrate this point, the geometric muscle model of the musculoskeletal shoulder model presented in this paper is based on the wrapping framework (number of fibres and types of wrapping surfaces) from the VHP model. The geometric muscle model of the VHP shoulder model was constructed using the centroid line approach and the guidelines to model muscles with large attachment sites (van der Helm and Veenbaas 1991). Implementations of the VHP model have shown no activation of the DLTa and no solution to the musculo-tendon force coordination problem in certain configurations of the model during simple movements such as abduction and elevation (Ingram et al. 2012; Quental et al. 2015). Therefore, using the minimal number of ideal fibres, placed at the centroids, does not guarantee an adequate representation of the musculature. The theoretical result regarding 
muscles with large attachment sites defines a necessary condition of the ideal muscle fibre model. The momentarm matrix must be full column rank. This result is necessary to invert the torque-force map, used as an equality constraint, but is not sufficient to guarantee the existence of a solution to the musculo-tendon force coordination problem. The imposed actuation vector must be inside the torque-feasibility space. This occurs only if the desired kinematics lie inside the WFS. There are algorithms for computing the WFS (Bosscher et al. 2006; Gouttefarde et al. 2011) but they are highly non-trivial. An improved approach over current methods of building models of the musculature using ideal fibres is to use an optimal fibre placement algorithm. Such an algorithm would iterate on the topology of each fibre to obtain the best possible load-bearing capabilities. The muscle fibre wrench $\mathscr{F}_{Q}$ (cf. Section 2.3) would link fibre topology to the solution space of the muscle coordination problem. The algorithms developed to analyse a model's ability to bear loads could be used to assess the topology at each iteration. However, the development of optimal fibre topologies remains a challenging task.

To conclude, the analysis presented in this paper is a preliminary analysis, used to locally (only two muscles) improve the geometric muscle model of a musculoskeletal shoulder model. It showed that moment-arms are the key element and govern the solution set through the TFS. The analysis of the TFS could be used inside an optimisation scheme to globally improve the topology of the ideal muscle fibre model, yielding more realistic estimates of musculo-tendon forces.

\section{Funding}

This study was supported by the Swiss National Science Foundation [grant number K-32K1-122512].

\section{Conflict of interest disclosure statement}

No potential conflict of interest was reported by the authors.

\section{Note}

1. A polytope is a geometric object with flat sides. Examples include polygons in two dimensions and polyhedrons in three dimensions.

\section{References}

Aeberhard M, Michellod Y, Mullhaupt P, Terrier A, Pioletti D, Gillet D. 2009. Dynamical biomechanical model of the shoulder: Null space based optimization of the overactuated system. In: IEEE international conference on robotics and biomimetics (ROBIO) 2008. February. p. 67-73.

Audenaert A, Audenaert E. 2008. Global optimization method for combined spherical-cylindrical wrapping in musculoskeletal upper limb modelling. Computer Methods Programs Biomed. 92(1):8-19. doi:10.1016/j.cmpb.2008.05.005.
Ball SR. 1876. Theory of Screws: a study in the dynamics of a rigid body. Dublin: Hodges Publication.

Baumgarte J. 1983. A new method of stabilization for holonomic constraints. J Appl Mech. 50(4a):869-870. doi:10.1115/1. 3167159.

Bergmann G, Graichen F, Bender A, Kääb M, Rohlmann A, Westerhoff P. 2007. In vivo glenohumeral contact forcesmeasurements in the first patient 7 months postoperatively. J Biomech. 40(10):2139-2149. doi:10.1016/j.jbiomech. 2006.10.037.

Bosscher P, Riechel A, Ebert-Uphoff I. 2006. Wrench-feasible workspace generation for cable-driven robots. Rob IEEE Trans. 22(5):890-902. doi:10.1109/TRO.2006.878967.

Klein Breteler MD, Spoor C, van derHelm F. 1999. Measuring muscle and joint geometry parameters of a shoulder for modeling purposes. J Biomech. 32(11):1191-1197. doi:10. 1016/S0021-9290(99)00122-0.

Carman A, Milburn P. 2005. Dynamic coordinate data for describing muscle-tendon paths: a mathematical approach. J Biomech. 38(4):943-951. doi:10.1016/j.jbiomech.2004. 03.017.

Charlton I, Johnson G. 2006. A model for the prediction of the forces at the glenohumeral joint. In: Proceedings of the institution of mechanical engineers $[\mathrm{H}]$. 220. p. 801-812. doi:10.1243/09544119JEIM147.

Chu A, Hughes R. 2010. A method to determine whether a musculoskeletal model can resist arbitrary external loadings within a prescribed range. Comput Methods Biomech Biomed Eng. 13(6):795-802. doi:10.1080/102558410 03630629.

Damsgaard M, Rasmussen J, Christensen S, Surma E, De Zee M. 2006. Analysis of musculoskeletal systems in the AnyBody Modeling System. Simul Model Pract Theory. 14(8): 1100-1111. doi:10.1016/j.simpat.2006.09.001.

Delp S, Loan J, Hoy M, Zajac F, Topp E, Rosen J. 1990. An interactive graphics-based model of the lower extremity to study orthopaedic surgical procedures. IEEE Trans Biomed Eng. 37(8):757-767. doi:10.1109/10.102791.

Desailly E, Sardain P, Khouri N, Yepremian D, Lacouture P. 2010. The convex wrapping algorithm: a method for identifying muscle paths using the underlying bone mesh. J Biomech. 43(13):2601-2607. doi:10.1016/j.jbiomech. 2010.05.005.

Dickerson C, Chaffin D, Hughes R. 2007. A mathematical musculoskeletal shoulder model for proactive ergonomic analysis. Comput Methods Biomech Biomed Eng. 10(6):389-400. doi:10.1080/10255840701592727.

El Habachi A, Duprey S, Cheze L, Dumas R. 2014. Springer Netherlands; a parallel mechanism of the shoulderapplication to multi-body optimisation. Multibody Sys Dyn. 33(4):1-13.

Engelhardt C, Ingram D, Muellhaupt P, Pralong E, Farron A, Pioletti D, Terrier A. 2012. Solving overconstrained kinematic in numerical shoulder model using nullspace optimization. In: 10th international symposium on computer methods in biomechanics and biomedical engineering, (CMBBE) 2012 Berlin. April.

Erdemir A, McLean S, Herzog W, van denBogert A. 2007. Model-based estimation of muscle forces exerted during movements. Clin Biomech. 22(2):131-154. doi:10.1016/j. clinbiomech.2006.09.005

Escamilla R, Yamashiro K, Paulos L, Andrews J. 2009. Shoulder muscle activity and function in common shoulder rehabilitation exercises. Sports Med. 39(8):663-685. doi:10. 2165/00007256-200939080-00004. 
Favre P, Snedeker J, Gerber C. 2009. Numerical modelling of the shoulder for clinical applications. Philos Trans Ser A Math Phys Eng Sci. 367(1895):2095-2118. doi:10.1098/rsta.2008.0282.

Garner B, Pandy M. 1999. A kinematic model of the upper limb based on the visible human project (VHP) image dataset. Comput Methods Biomech Biomed Eng. 2(2):107-124. doi:10.1080/10255849908907981.

Garner B, Pandy M. 2000. The obstacle-set method for representing muscle paths in musculoskeletal models. Comput Meth Biomech Biomed Eng. 3(1):1-30. doi:10. $1080 / 10255840008915251$

Garner B, Pandy M. 2001. Musculoskeletal model of the upper limb based on the visible human male dataset. Comput Methods Biomech Biomed Eng. 4(2):93-126. doi:10.1080/ 10255840008908000 .

Gouttefarde M, Merlet JP, Daney D. 2007. Wrench-feasible workspace of parallel cable-driven mechanisms. In: IEEE international conference on robotics and automation, 2007. p. $1492-1497$.

Gouttefarde M, Daney D, Merlet JP. 2011. Interval-analysisbased determination of the wrench-feasible workspace of parallel cable-driven robots. IEEE Trans Rob. 27(1):1-13. doi:10.1109/TRO.2010.2090064.

Grunbaum B. 2003. Convex Polytopes. 2nd ed. New York, NY: Spinrger-Verlag.

Hand L, Finch J. 1998. Analytical Mechanics. New York, NY: Cambridge University Press.

Holzbaur KRS, Murray WM, Delp SL. 2005. A model of the upper extremity for simulating musculoskeletal surgery and analyzing neuromuscular control. Ann Biomed Eng. 33(6):829-840. doi:10.1007/s10439-005-3320-7.

Högfors C, Peterson B, Herberts P. 1987. Biomechanical model of the human shoulder-I. Elements. J Biomech. 20(2): 157-166.

Högfors C, Peterson B, Sigholm G, Herberts P. 1991. Biomechanical model of the human shoulder-II. The shoulder ryrthm. J Biomech. 24(8):699-709.

Ingram D, Müllhaupt P, Terrier A, Pralong E, Farron A. 2012. Dynamical biomechanical model of the shoulder for muscle-force estimation. In: IEEE international conference on biomedical robotics and biomechatronics (BIOROB) 2012. June.

Ingram D, Engelhardt $\mathrm{C}$, Farron A, Terrier A, Müllhaupt $\mathrm{P}$. 2013a. A minimal set of coordinates for describing humanoid shoulder motion. In: IEEE/RSJ international conference on intelligent robots and systems (IROS), 2013. p. $5537-5544$.

Ingram D, Engelhardt C, Farron A, Terrier A, Müllhaupt P. 2013b. Muscle moment-arms: a key element in muscle-force estimation. Comput Methods Biomech Biomed Eng. :1-8.

Karlsson D, Peterson B. 1992. Towards a model for force predictions in the human shoulder. J Biomech. 25(2):189-199. doi:10.1016/0021-9290(92)90275-6.

Lemieux P, Hagemeister N, Tétreault P, Nuño. 2013. Influence of the medial offset of the proximal humerus on the glenohumeral destabilising forces during arm elevation: a numerical sensitivity study. Comput Methods Biomech Biomed Eng. 16(1):103-111. doi:10.1080/10255842.2011. 607813.

Makhsous M, Högfors C, Siemien'ski A, Peterson B. 1999. Total shoulder and relative muscle strength in the scapular plane. J Biomech. 32(11):1213-1220. doi:10.1016/S0021-9290 (99)00049-4.

Masjedi M, Johnson G. 2010. Glenohumeral contact forces in reversed anatomy shoulder replacement.
J Biomech. 43(13):2493-2500. doi:10.1016/j.jbiomech. 2010.05.024.

Nikooyan A, Veeger H, Westerhoff P, Graichen F, Bergmann G, van derHelm F. 2010. Validation of the Delft Shoulder and Elbow Model using in-vivo glenohumeral joint contact forces. J Biomech. 43(15):3007-3014. doi:10.1016/j.jbiomech.2010.06.015.

Nof S. 1999. Handbook of industrial robotics. Vol. 1. New York, NY: John Wiley \& Sons.

Poinsot ML. 1848. The elements of statics. (Translated from French). Cambridge: Cambridge University Press.

Poppen N, Walker P. 1978. Forces at the glenohumeral joint in abduction. J Clin Orthopedics. 135:165-170.

Prinold J, Masjedi M, Johnson G, Bull A. 2013. Musculoskeletal shoulder models: a technical review and proposals for research foci. J Eng Med. 227(10):1041-1057. doi:10.1177/ 0954411913492303.

Quental C, Folgado J, Ambrósio J, Monteiro J. 2012. A multibody biomechanical model of the upper limb including the shoulder girdle. Multibody Sys Dyn. 28(1-2):83-108. doi:10.1007/s11044-011-9297-0.

Quental C, Folgado J, Ambrósio J, Monteiro J. 2015. Critical analysis of musculoskeletal modelling complexity in multibody biomechanical models of the upper limb. Comput Methods Biomech Biomed Eng. 18(7):749-759. doi:10. 1080/10255842.2013.845879.

Raikova R. 1992. A general approach for modelling and mathematical investigation of the human upper limb. J Biomech. 25(8):857-867. doi:10.1016/0021-9290(92)90226-Q.

Rasmussen J, Damsgaard M, Voigt M. 2001. Muscle recruitment by the $\mathrm{min} / \mathrm{max}$ criterion - a comparative numerical study. J Biomech. 34(3):409-415. doi:10.1016/S0021-9290(00) 00191-3.

Seireg A, Arvikar R. 1973. A mathematical model for evaluation of forces in lower extremeties of the musculo-skeletal system. J Biomech. 6(3):313-326. doi:10.1016/0021-9290 (73)90053-5.

Sins L. 2014. Experimental and numerical study of anatomical total joint replacement of the shoulder École de Technologie Supérieure. Montréal, Canada.

Sternad D. 2008. Progress in motor control: a multidisciplinary perspective. New York, NY: Springer Science \& Business Media.

Terrier A, Aeberhard M, Michellod Y, Mullhaupt P, Gillet D, Farron A, Pioletti D. 2010. A musculoskeletal shoulder model based on pseudo-inverse and null-space optimization. Medical Eng Phys. 32(9):1050-1056. doi:10.1016/j. medengphy.2010.07.006.

Terrier A, Reist A, Merlini F, Farron A. 2008. Simulated joint and muscle forces in reversed and anatomic shoulder prostheses. J Bone Joint Surg. 90-B(6):751-756. doi:10. 1302/0301-620X.90B6.19708.

Valero-Cuevas F, Anand V, Saxena A, Lipson H. 2007. Beyond parameter estimation: extending biomechanical modeling by the explicit exploration of model topology. IEEE Trans Biomed Eng. 54(11):1951-1964. doi:10.1109/TBME.2007. 906494.

Valero-Cuevas F, Zajac F, Burgar C. 1998. Large index-fingertip forces are produced by subject-independent patterns of muscle excitation. J Biomech. 31(8):693-703. doi:10.1016/ S0021-9290(98)00082-7.

van der Helm F. 1994a. A finite element musculoskeletal model of the shoulder mechanism. J Biomech. 27(5):551-569. doi:10.1016/0021-9290(94)90065-5. 
van der Helm F. 1994b. Analysis of the kinematic and dynamic behavior of the shoulder mechanism. J Biomech. 27(5):527-550. doi:10.1016/0021-9290(94)90064-7.

van der Helm F, Veenbaas R. 1991. Modelling the mechanical effect of muscles with large attachment sites: application to the shoulder mechanism. J Biomech. 24(12):1151-1163. doi:10.1016/0021-9290(91)90007-A.

Webb J. 2011. Contributions of the deltoid and rotator cuff to shoulder mobility and stability: a 3D finite element analysis. Stanford, CA: Stanford University.

Wu G, van der Helm FCT, (DirkJan) Veeger HEJ, Makhsous M, Van Roy P, Anglin C, Nagels J, Karduna AR, McQuade K,
Wang X, et al. 2005. ISB recommendation on definitions of joint coordinate systems of various joints for the reporting of human joint motion-Part II: shoulder, elbow, wrist and hand. J Biomech. 38(5):981-992. doi:10.1016/j.jbiomech. 2004.05.042.

Yanagawa T, Goodwin C, Shelburne K, Giphart J, Torry M, Pandy M. 2008. Contributions of the individual muscles of the shoulder to glenohumeral joint stability during abduction. J Biomech Eng. 130(2):1-9.

Yang J, Feng X, Kim J, Rajulu S. 2010. Review of biomechanical models for human shoulder complex. Int J Hum Factors Simul. 1(3):271-293. doi:10.1504/IJHFMS.2010.036791. 